\title{
Exploring the Proton Spin with Di-jets at a Future EIC
}

\section{Brian Page*}

Brookhaven National Laboratory

E-mail: bpageabnl . gov

\begin{abstract}
Since the memorable results from the EMC collaboration nearly thirty years ago showed that the valence quark contribution to the spin of the proton was consistent with zero, the question of how the proton spin arises from the intrinsic spin and orbital angular momenta of its constituents has vexed the scientific community. Despite significant experimental and theoretical progress, it is clear that current facilities lack the precision and kinematic range needed to fully address this question. A proposed high-luminosity Electron-Ion Collider (EIC) with polarized electron and proton beams will have the capability to pin down the intrinsic quark and gluon contributions as well as address the orbital angular momentum. Measurements of the $g_{1}$ structure function and its scaling violation will be the golden channel for probing the quark and gluon spins. The gluon can also be accessed by tagging photon-gluon fusion events using dijet final states. This talk will give an overview of the dijet measurement as well as discuss general issues in jet finding at an EIC.
\end{abstract}

XXV International Workshop on Deep-Inelastic Scattering and Related Subjects

3-7 April 2017

University of Birmingham, $U K$

\footnotetext{
*Speaker.
} 


\section{Introduction}

In the thirty years since the EMC collaboration showed that the spin of the proton cannot be built up exclusively from the spins of its constituent quarks [1], much progress has been made in understanding the helicity structure of the proton. Subsequent fixed-target polarized deep inelastic scattering (DIS) experiments [2] have shown that roughly a third of the proton's spin is due to the intrinsic spins of the quarks and antiquarks in the Bjorken- $x\left(x_{B}\right)$ region greater than $10^{-3}$ [3]. Additionally, recent results from the Relativistic Heavy Ion Collider (RHIC) [4, 5] have been incorporated into next-to-leading order (NLO) global analyses that for the first time find evidence for a non-zero gluon polarization in the range $x_{B}>0.05[6,7]$.

Despite these results, a detailed understanding of the origin of the proton spin remains incomplete. While the polarized DIS and RHIC results place strong constraints on the quark and gluon helicity distributions in their respective regions of sensitivity, uncertainties at lower values of $x_{B}$ remain sizable. In addition, the contribution from parton orbital angular momentum is virtually unconstrained by data. While ongoing measurements at RHIC aim to extend constraints on the gluon polarization to lower $x_{B}$ values by utilizing higher center-of-mass energies and final states at more forward rapidities, it is clear that current facilities lack the kinematic reach to fully answer the fundamental question of how the proton gets its spin.

In order to resolve this question, a new facility will be required which has the ability to reach low values of $x_{B}$ as well as precisely determine the event kinematics. These conditions will be realized in a proposed electron-ion collider (EIC).

\section{Event Selection and Kinematics}

The golden channel for the determination of the gluon helicity distribution, $\Delta g\left(x, Q^{2}\right)$, at an EIC will be the measurement of the $Q^{2}$ dependence of the $g_{1}$ structure function. The expanded kinematic reach available at a collider will provide the large lever-arm in $Q^{2}$ needed to accurately determine $\Delta g\left(x, Q^{2}\right)$. These $g_{1}$ measurements are not the only way to access gluon information in DIS, however. The photon-gluon fusion (PGF) process, in which a gluon splits into a quarkantiquark pair that subsequently interacts with the exchanged virtual photon, is also sensitive to the gluon. The PGF reaction produces a pair of azimuthally separated high- $p_{\mathrm{T}}$ partons which hadronize to form a dijet. These proceedings outline a method utilizing dijets to select PGF events as well as determine the initial gluon kinematics. Sensitivity to $\Delta g\left(x, Q^{2}\right)$ is then obtained via measurements of the longitudinal double-spin asymmetry, $A_{L L}$. For this study, DIS events were generated using PYTHIA 6.4 [8] and stable particles with transverse momenta above $250 \mathrm{MeV} / c$ were clustered into jets (in the Breit frame) using the anti- $\mathrm{k}_{T}$ algorithm [9] as implemented in the FastJet package [10]. Dijets were selected by finding the two jets with highest transverse momenta $\left(p_{T}\right)$ and requiring that one jet has $p_{T}>5 \mathrm{GeV} / c$ and the other $p_{T}>4 \mathrm{GeV} / c$ while being greater than $120^{\circ}$ apart in azimuthal angle.

In addition to the PGF subprocess, dijets can arise from QCD-Compton (QCDC), resolved, and even the leading-order DIS subprocesses, which constitute a background to the gluon measurements. It is particularly important to reduce the contribution from the resolved subprocess, in which the photon behaves hadronically and contributes a quark or gluon to the hard scattering, as 

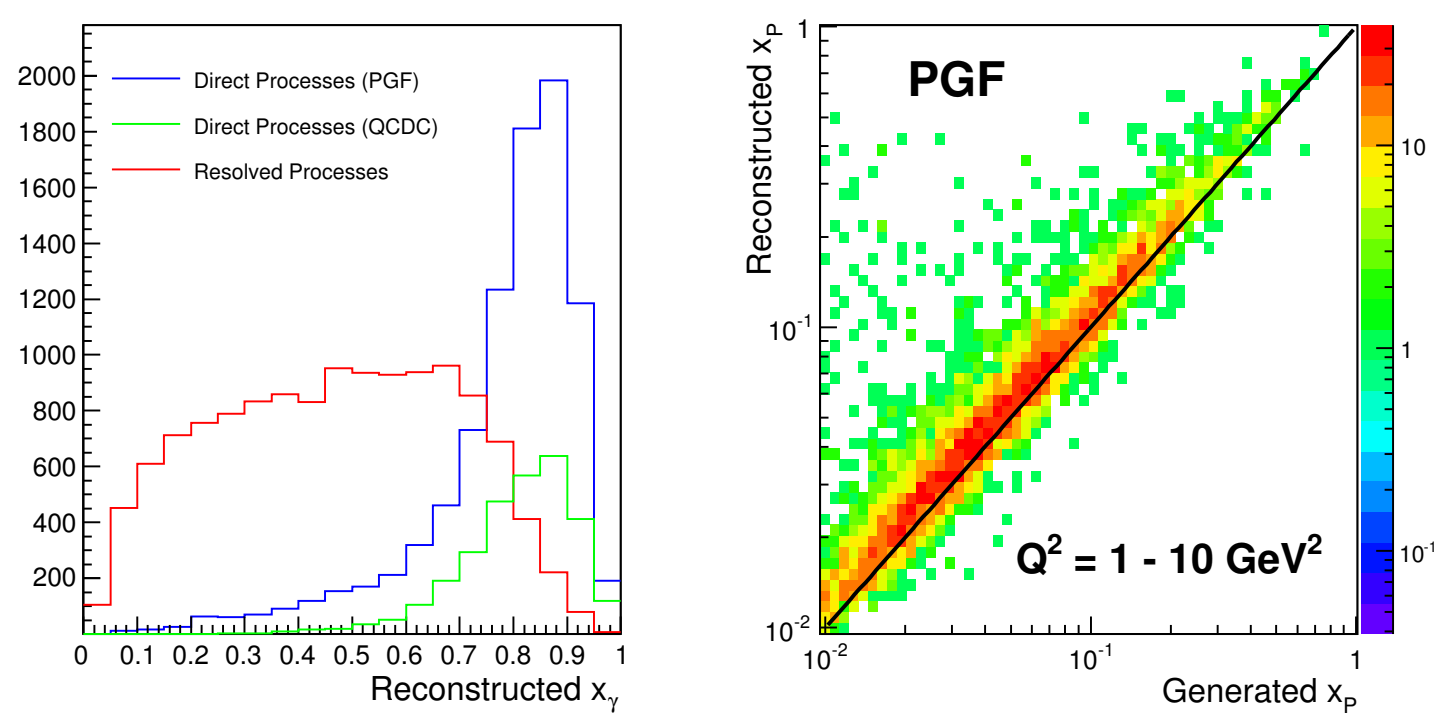

Figure 1: (Left) Yields of events from PGF, QCDC, and resolved subprocesses plotted as a function of reconstructed $x_{\gamma}$. (Right) Comparison of reconstructed to generated $x_{P}$ for PGF events with $Q^{2}=1-10$ $\mathrm{GeV}^{2}$ and $x_{\gamma}>0.8$.

the asymmetry for this subprocess depends on the polarized photon parton distribution functions (PDFs) which are completely unconstrained. It should be noted that there is a proposal to measure the polarized photon PDFs using a method very similar to that which is discussed below.

To reduce contributions from resolved subprocesses, the dijet kinematics are used to reconstruct the momentum fraction of the photon contribution to the reaction, denoted $x_{\gamma}$, according to the formula:

$$
x_{\gamma}=\frac{1}{2 \mathrm{E}_{e} y}\left(m_{T_{1}} \mathrm{e}^{-Y_{1}}+m_{T_{2}} \mathrm{e}^{-Y_{2}}\right) .
$$

Here $\mathrm{E}_{e}$ refers to the electron beam energy, $y$ is the inelasticity, $m_{T_{1,2}}$ are the transverse masses $\left(\sqrt{m^{2}+p_{\mathrm{T}}^{2}}\right)$ of the two jets, and $Y_{1,2}$ are the rapidities of the two jets as measured in the laboratory frame. Because the virtual photon interacts as a point-like particle for PGF and QCDC, $x_{\gamma}$ for these subprocesses should be unity. On the other hand, $x_{\gamma}$ for the resolved subprocess can take on a range of values representing the momentum fraction carried by the parton that participates in the scattering. This can be seen in the left panel of Fig. 1 which shows the yield of dijets from each subprocess plotted versus $x_{\gamma}$, given by Eq. 2.1, for $Q^{2}$ between 1 and $10 \mathrm{GeV}^{2}$ where the PGF and QCDC curves are peaked at high $x_{\gamma}$ values. For the results presented below, events with $x_{\gamma}$ below 0.8 are dropped, removing a large fraction of resolved events.

In addition to $x_{\gamma}$, the momentum fraction of the struck parton from the proton $\left(x_{P}\right)$ can be determined via an equation similar to Eq. 2.1 with the term $\mathrm{E}_{e} y$ replaced with the proton beam energy and $\mathrm{e}^{-Y_{1,2}}$ replaced with $\mathrm{e}^{+Y_{1,2}}$. The good agreement between reconstructed and generated $x_{P}$ can be seen in the right panel of Fig. 1. An alternative expression, which relates $x_{P}$ with 
Bjorken-x, the dijet mass $M$, squared center-of-mass collision energy $s$, and inelasticity $y$ is given by the formula:

$$
x_{P}=x_{B}\left(1+\frac{M^{2}}{Q^{2}}\right)=x_{B}+\frac{M^{2}}{s y} .
$$

This expression makes explicit that the lowest accessible $x_{P}$ at an EIC is roughly $5 \times 10^{-3}$, with $M=10 \mathrm{GeV}, y=0.95$, and $s=20000 \mathrm{GeV}^{2}$, corresponding to electron and proton beam energies of 20 and $250 \mathrm{GeV}$, respectively. It can also be seen that a given dijet mass range will be sensitive to a particular $x_{P}$ range, largely independent of $Q^{2}$. The ability to select the same $x_{P}$ over a wide $Q^{2}$ range will allow for investigations of the evolution of $\Delta g\left(x, Q^{2}\right)$.

\section{Expected Asymmetry}

As mentioned above, access to the gluon polarization in this study will come from measuring the longitudinal double-helicity asymmetry $A_{L L}$. To determine the size of the required dataset and the level to which systematic effects will need to be controlled, an estimation of the size of the expected asymmetry is needed. In order to introduce an asymmetry in the PYTHIA simulation, the events were reweighted following a procedure used by the HERMES collaboration in the extraction of $\Delta G$ from their high- $p_{T}$ charged hadron data [11]. Each event was assigned a weight according to:

$$
w=\hat{a}\left(\hat{s}, \hat{t}, \mu^{2}, Q^{2}\right) \frac{\Delta f_{a}^{\gamma^{*}}\left(x_{a}, \mu^{2}\right)}{f_{a}^{\gamma^{*}}\left(x_{a}, \mu^{2}\right)} \frac{\Delta f_{b}^{N}\left(x_{b}, \mu^{2}\right)}{f_{b}^{N}\left(x_{b}, \mu^{2}\right)},
$$

where $\hat{a}\left(\hat{s}, \hat{t}, \mu^{2}, Q^{2}\right)$ is the subprocess dependent parton level asymmetry, $(\Delta) f_{a}^{\gamma^{*}}\left(x_{a}, \mu^{2}\right)$ is the (polarized) PDF for the virtual photon, and $(\Delta) f_{b}^{N}\left(x_{b}, \mu^{2}\right)$ is the (polarized) PDF for the proton. The expressions for $\hat{a}$ are calculable and can be found in [11] while the PDF sets used for the unpolarized proton, polarized proton, and unpolarized photon distributions were CTEQ5M [12], DSSV14 [6], and GRV-G [13], respectively. Since polarized photon PDFs are not available, we use the so-called maximal scheme in which the polarized PDF is set equal to the unpolarized PDF at an input scale. Note that for direct subprocesses, the polarized over unpolarized photon PDF ratio is identically unity.

With the event-by-event weights applied, the asymmetry is given by the average over the weights. The statistical uncertainties on $A_{L L}$ were determined using the formula:

$$
\sigma=\sqrt{\frac{1}{\mathrm{~N}}-\frac{A_{L L}^{2}}{\mathrm{~N}}}
$$

where $\mathrm{N}$ is the number of expected events assuming $10 \mathrm{fb}^{-1}$ of integrated luminosity. A factor accounting for the beam polarizations was not included in the determination of the uncertainty, but assuming proton and electron polarizations of $70 \%$ and $80 \%$, respectively, the uncertainties would grow by a factor of approximately 1.3 .

As can be seen in the left panel of Fig. 2, the magnitudes of the asymmetries for the QCDC and PGF subprocesses grow with dijet mass and become quite sizable. However, the QCDC and PGF 

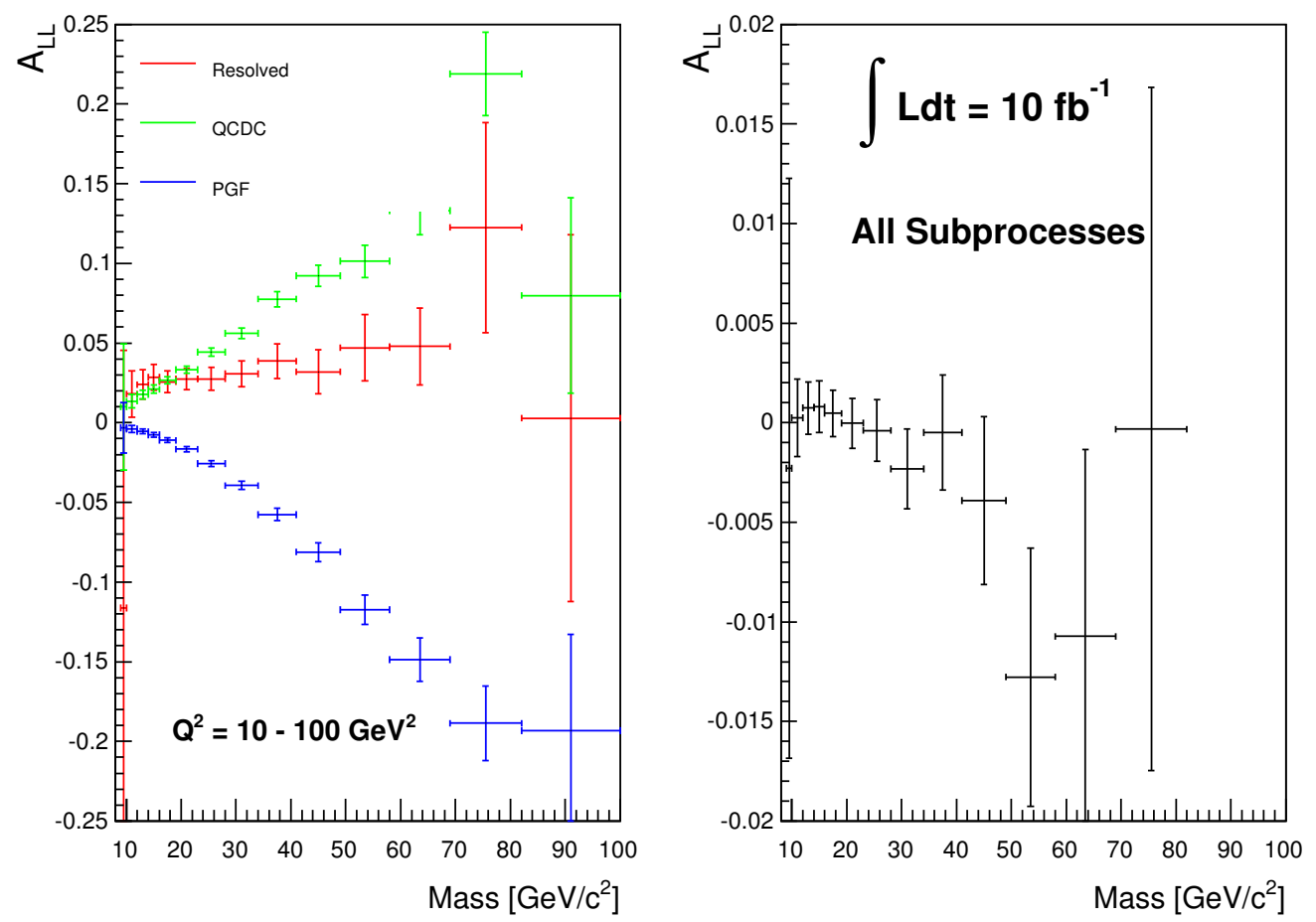

Figure 2: (Left) $A_{L L}$ versus dijet invariant mass for the photon-gluon fusion, QCD-Compton, and resolved subprocesses. (Right) Combined $A_{L L}$ from all subprocesses for $Q^{2}$ between 10 and $100 \mathrm{GeV}^{2}$.

asymmetries have opposite signs and nearly equal magnitudes, yielding a combined asymmetry that is quite small, as shown in the right panel. Similar cancellation can be seen in Fig. 3, which shows the asymmetry as a function of $x_{P}$ for dijet masses between 10 and $20 \mathrm{GeV} / c^{2}$. While the size of the PGF asymmetry is quite small over all $x_{P}$ in this dijet mass range, the PGF cross section at small $x_{P}$ is much larger than that for the QCDC subprocess. This gives rise to a negative asymmetry at low $x_{P}$ that becomes positive at larger $x_{P}$ where QCDC events are more prevalent. Measuring such small asymmetries will be experimentally challenging and control of systematics will be paramount. The cancellation between the QCDC and PGF asymmetries also argues for further study into suppressing QCDC events.

\section{Summary}

A method for probing the gluon polarization, $\Delta g\left(x, Q^{2}\right)$, at a future electron-ion collider using dijet final states was presented. Utilizing dijets as a surrogate for the hard-scattered partons, a method for efficiently rejecting resolved subprocess events was demonstrated and the ability to reconstruct accurately the struck parton momentum was verified. The magnitude of the expected asymmetry was found using a reweighting procedure and determined to be small. Further work on this topic will include investigations into reducing the contribution from QCDC events in order to enhance sensitivity to the gluon polarization, and introducing realistic detector smearing effects to better determine how well the partonic kinematics can be reconstructed experimentally. 

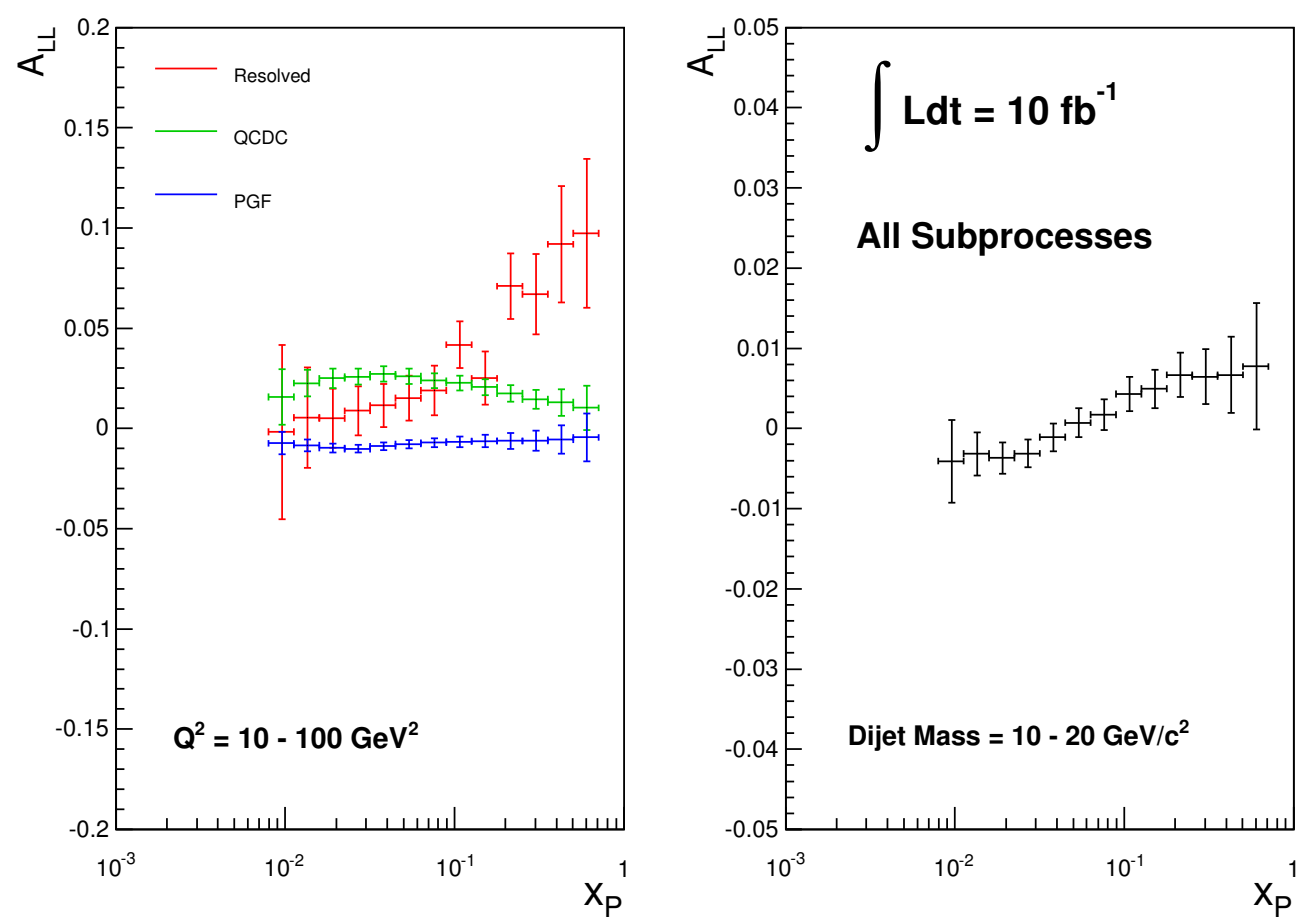

Figure 3: (Left) $A_{L L}$ versus reconstructed $x_{P}$ for the photon-gluon fusion, QCD-Compton, and resolved subprocesses for dijet invariant masses between 10 and $20 \mathrm{GeV} / c^{2}$. (Right) Combined $A_{L L}$ from all subprocesses for $Q^{2}$ between 10 and $100 \mathrm{GeV}^{2}$.

\section{References}

[1] J. Ashman et al. [European Muon Collaboration], Nucl. Phys. B 328, 1 (1989).

[2] C. A. Aidala, S. D. Bass, D. Hasch, and G. K. Mallot, Rev. Mod. Phys. 85, 655 (2013); and references therein.

[3] D. de Florian, R. Sassot, M. Stratmann, and W. Vogelsang, Phys. Rev. Lett. 101, 072001 (2008); Phys. Rev. D 80, 034030 (2009).

[4] L. Adamczyk et al. [STAR Collaboration], Phys. Rev. Lett. 115, 092002 (2015).

[5] A. Adare et al. [PHENIX Collaboration], Phys. Rev. D 90, 012007 (2014)

[6] D. de Florian, R. Sassot, M. Stratmann and W. Vogelsang, Phys. Rev. Lett. 113, 012001 (2014)

[7] E. R. Nocera et al. [NNPDF Collaboration], Nucl. Phys. B 887, 276 (2014)

[8] T. Sjostrand, S. Mrenna, and P. Z. Skands, JHEP 05, 026 (2006).

[9] M. Cacciari, G. P. Salam, and G. Soyez, JHEP 04, 063 (2008).

[10] M. Cacciari, G. P. Salam, and G. Soyez, Eur. Phys. J. C 72, 1896 (2012).

[11] A. Airapetian et al. [HERMES Collaboration], JHEP 1008, 130 (2010)

[12] H. L. Lai et al. [CTEQ Collaboration], Eur. Phys. J. C 12, 375 (2000)

[13] M. Gluck, E. Reya and A. Vogt, Phys. Rev. D 48, 116 (1993) 